\title{
Impact des facteurs individuels et environnementaux sur le taux d'aberrations chromosomiques de type translocations Partie 1 : âge, sexe, tabac, alcool
}

\author{
E. GRÉGOIRE ${ }^{1}$, G. GRUEL ${ }^{1}$, C. MARTIN ${ }^{1}$, S. ROCH-LEFÈVRE ${ }^{1}$, \\ A. VAURIJOUX ${ }^{1}$, P. VOISIN ${ }^{1}$, L. ROY $^{1}$
}

(Manuscrit reçu le 13 mai 2009, accepté le 14 décembre 2009)

RÉSUMÉ L'évaluation de l'exposition aux rayonnements ionisants, effectuée longtemps après l'exposition, est actuellement réalisée en dénombrant les aberrations chromosomiques de type translocations. Le taux de ces translocations observées dans les lymphocytes des personnes exposées est comparéau taux observé au sein d'une population contrôle. Toutefois, la spécificité des translocations vis-à-vis de l'irradiation n'est pas clairement identifiée. Afin d'éviter toute conclusion hâtive, il est nécessaire d'identifier tous les facteurs susceptibles d'induire des translocations. À notre connaissance, aucune synthèse sur l'effet de ces différents facteurs sur le taux de translocations n'a été réalisée à ce jour. Cette recherche bibliographique a confirmé l'impact de certains facteurs personnels sur l'augmentation des translocations. Cette étude corrobore que l'âge s'avère être le facteur ayant le plus d'impact sur le taux de translocations, notamment après 60 ans. À ce jour, le facteur « âge » est déjà considéré dans l'estimation du taux de translocations après suspicion d'exposition aux rayonnements ionisants pour toutes les classes d'âge. L'étude montre également que ce taux varie significativement lorsque le patient est exposé simultanément et de manière importante et chronique à une combinaison alcool et tabac. Ainsi, une courbe du taux de translocations devrait être établie en fonction de la consommation excessive de ce type d'agent pour chaque individu. Ainsi il serait alors possible de déterminer le taux de translocations induit uniquement par une exposition radiologique. Les effets des agents toxiques sur le taux de translocations après exposition professionnelle feront l'objet d'une deuxième partie.

ABSTRACT Impact of personal and environmental factors on the rate of chromosome aberrations named translocations - Part 1: age, gender, smoking, alcohol.

The assessment of exposure to ionizing radiation, carried out long time after exposure, is currently performed by scoring of translocations, a specific type of chromosomal aberrations. The translocations rate observed in peripheral blood lymphocytes of exposed subjects is compared to that observed in a control population. However, the translocation specificity towards radiation exposure is not clearly identified. To avoid any hasty conclusion, it is necessary to identify all the factors likely to induce translocation. To our knowledge, no study has thus far examined the effects of all these different factors on translocation rates. A review of the literature thus allowed us to assess the impact of host factors and lifestyle on the production of translocations. This study confirms that age appears to be the factor

\footnotetext{
1 IRSN, Laboratoire de Dosimétrie Biologique, BP 17, 92262 Fontenay-aux-Roses Cedex, France.
} 
having the greatest impact on the rate of translocations, especially over 60 years. To date, the factor "age" is already considered in estimating the impact of radiation on the rate of translocation for all age groups. However, the study also shows that this rate varies significantly when the patient is exposed simultaneously and significantly towards many lifestyle agents. A precise threshold translocation rate should thus be established as a function of known behavioral exposures, below which it is impossible to conclude that radiological exposure has occurred. The effects of chemicals on the translocation rate after occupational exposure will be the subject of a second part.

Keywords: Chromosomal translocation / lifestyle factor / individual agent / retrospective dosimetry / environmental factor

\section{Introduction}

L'évaluation d'une exposition récente aux rayonnements ionisants à l'aide d'un marqueur biologique est aujourd'hui une technique fiable et parfaitement codifiée. La méthode utilisée est fondée sur le dénombrement des anomalies chromosomiques radio-induites (Fig. 1), le dicentrique étant l'aberration chromosomique de choix dans ce cas.

Néanmoins, la méthode de dénombrement des dicentriques se révèle être inadaptée à l'estimation d'expositions anciennes car ce type d'aberrations chromosomiques radio-induites n'est pas stable dans le temps. En effet, de part sa configuration, la présence de 2 centromères crée un pont lors de la division cellulaire et par conséquent empêche la cellule de se diviser. De ce fait, les cellules porteuses de dicentriques disparaissent à chaque division cellulaire et ainsi l'information concernant l'estimation de la dose d'irradiation diminue considérablement 1 an après l'exposition. Or, l'expérience acquise par le Laboratoire de dosimétrie biologique de l'IRSN atteste une demande croissante de l'évaluation dosimétrique plusieurs années après l'exposition. En effet, les personnes ayant pu être surexposées professionnellement, ou affirmant avoir été exposées accidentellement souhaitent, par prescription médicale, avoir un bilan dosimétrique de leur exposition. La dosimétrie biologique rétrospective peut permettre de répondre à de telles interrogations. Depuis une quinzaine d'années, une nouvelle technologie s'est développée, permettant de détecter facilement des anomalies chromosomiques relativement stables dans le temps. Ces aberrations très utiles en tant que marqueur d'expositions anciennes sont les translocations (Fig. 1). Même si quelques études ont montré une décroissance du taux de translocations avec le temps (Bauchinger et al., 2001 ; Lindholm et Edwards, 2004 ; Sorokine-Durm et al., 2000a ; Tucker, 2008), la stabilité des translocations est incontestablement meilleure que celle des dicentriques (Edwards et al., 2005 ; Grégoire et al., 2006 ; Lindholm et Edwards, 2004 ; Pala et al., 2001 ; Tawn et al., 2006 ; Tucker, 2008). L'étude de patients traités par radiothérapie durant des dizaines d'années pour spondylarthrite ankylosante, suggère que les translocations semblent persister plusieurs années après l'exposition (Buckton 


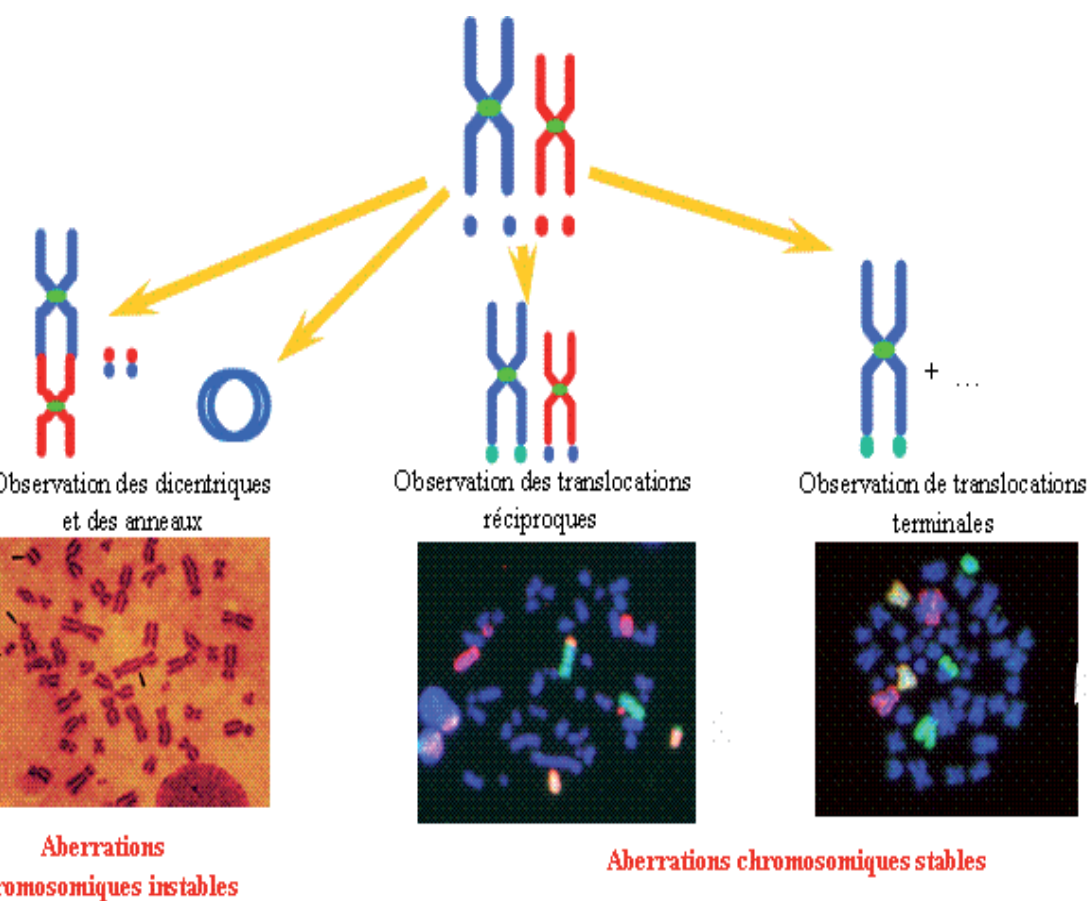

Figure 1 - Présentation des différents types d'aberrations chromosomiques observés en dosimétrie biologique.

Chromosomal aberrations observed in biological dosimetry.

et al., 1962). Une bonne stabilité du nombre de translocations a aussi été observée sur des singes irradiés in toto et suivis durant 3 ans (Grégoire et al., 2006). Des résultats similaires ont été relevés dans l'étude de suivi du taux de translocations pendant 4 ans chez des militaires Georgiens exposés aux rayonnements ionisants à Lilo (Roy et al., 2006).

Pour pouvoir réaliser une dosimétrie rétrospective, il est nécessaire de connaître, le taux de translocations en absence d'irradiation et donc la spécificité des translocations. Or ce taux n'étant pas nul, il conditionne en partie la sensibilité de la technique. Lors de l'estimation de la dose, le taux de translocations de la population non-exposée aux rayonnements ionisants doit être pris en compte afin de le confronter au taux de translocations dû à une exposition radiologique accidentelle. Il est donc nécessaire de connaître précisément ce bruit de fond ainsi que les facteurs susceptibles de le modifier. En effet, une méconnaissance des effets de l'exposition à de tels agents pourrait alors conduire à une surestimation 
de la dose de rayonnements ionisants reçue par la personne potentiellement exposée. Une revue de la production des aberrations chromosomiques par des facteurs individuels et liés aux habitudes de vie est nécessaire afin d'identifier leur capacité d'engendrer des aberrations chromosomiques au sein des cellules sanguines.

Parmi l'ensemble des études qui s'intéressent aux aberrations chromosomiques produites par ces facteurs, un faible nombre d'études caractérisent spécifiquement les translocations. Ainsi, les facteurs décrits dans ces études peuvent être répartis en 2 groupes :

- les facteurs individuels tels l'âge et le genre ;

- les facteurs liés aux habitudes de vie tels le tabac et l'alcool.

Pour les facteurs individuels, nous avons comparé les variations du taux de translocations de différentes classes d'âge, ainsi que celles observée chez les hommes et chez les femmes. Pour les habitudes de vie, nous nous sommes intéressés au taux de translocations du groupe contrôle et du ou des groupe(s) exposé(s). Ces 2 types de facteurs ont été étudiés sur le facteur d'augmentation des translocations et sur la différence significative ou non entre les taux des différents groupes.

\section{Matériels et méthodes}

La recherche bibliographique a été effectuée sur PubMed et Scopus à partir des mots-clés suivants: aberrations chromosomiques, facteurs carcinogènes ou mutagènes, translocation, et lymphocytes du sang circulant. Dans ces bases de données nous avons mentionné les mots clés en anglais suivants :

chromosom* aberration AND (blood OR "peripheral blood lymphocyte" OR "peripheral blood cell") AND (translocation)) AND NOT KEY((“ionizing radiation" OR "ionising radiation" OR radiotherapy OR radiation).

Sous PubMed :

AND (tobacco OR cigarette OR cigar OR "tobacco smoke" OR nicotine OR smoker OR alcohol OR ethanol OR alcoholic OR drug OR "drug addict*" OR "drug abuse" OR junkie OR junky OR cannabis OR heroin OR cocaine OR "life habit" OR dependence OR addiction OR "life style" OR "life style agent").

Sous Scopus :

AND (tobacco OR cigarette OR cigar OR "tobacco smoke" OR nicotine OR smoker OR alcohol OR ethanol OR alcoholic OR drug OR "drug addict*" OR "drug abuse" OR junkie OR junky). 

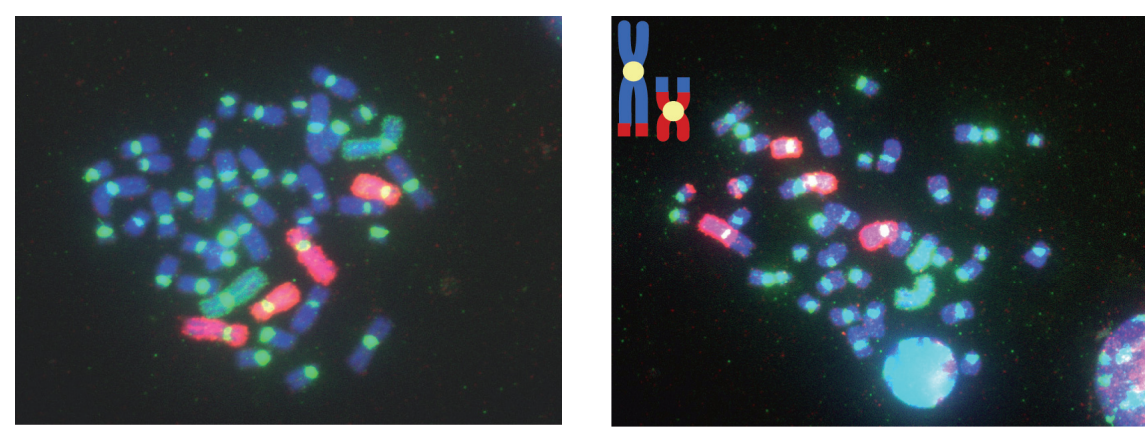

Figure 2-Marquage de 3 paires de chromosomes de lymphocytes sanguins par la technique FISH. À gauche, cellule normale; à droite, cellule présentant une translocation chromosomique entre des chromosomes peints de couleur différente.

Chromosome painting by the FISH technique. Left, normal blood lymphocyte; right, blood lymphocyte with chromosomal translocation between chromosomes painted in different colour.

Par conséquent, toutes les publications en rapport avec une exposition aux rayonnements ionisants, radiothérapie ou irradiation n'ont pas été prises en compte. Le nombre de publications traitant des effets de différents facteurs sur les translocations, fut assez faible. En effet, la plupart des publications ne font pas la distinction entre les aberrations chromosomiques stables (translocations) et instables (dicentriques), et ne font référence qu'à un taux global d'aberrations chromosomiques dit de structure. De telles publications n'ont pas été incluses dans cette étude car nous voulions identifier uniquement l'impact spécifique des agents étudiés sur la production des translocations. Ainsi, nous avons retenu une vingtaine de publications de 1982 à 2008 analysants les effets d'au moins un facteur sur le taux de translocations de lymphocytes d'individus exposés in vivo.

Pendant longtemps, l'analyse des translocations par la méthode conventionnelle a été difficile, nécessitant un caryotype complet de la cellule après coloration au Giemsa. L'introduction de la technique d'hybridation in situ fluorescente (FISH) a facilité cette analyse en permettant de «peindre » les chromosomes de couleurs différentes grâce à des sondes d'ADN spécifiques d'un chromosome donné et marquées par des molécules fluorescentes (Fig. 2). Dans la plupart des publications analysées, 3 chromosomes sont colorés (FISH 3 peintures), ce qui permet d'avoir accès seulement aux translocations impliquant les 3 chromosomes colorés. Tout échange de matériel entre les chromosomes peints et non peints est alors visualisé. Les différents laboratoires qui utilisent la technique FISH pour détecter les translocations ne marquent pas les mêmes chromosomes. Afin de pouvoir comparer les données entre elles, il est donc nécessaire d'estimer le taux de translocations 
présents sur l'ensemble du génome à partir des taux de translocations observés sur 3 chromosomes. Pour cela la formule de Lucas et al. (1992) est utilisée dans la majorité des publications analysées. Toutefois, pour certaines d'entre elles, il a fallu faire ce calcul quand le nombre de cellules analysées, le nombre de translocations et les chromosomes peints étaient indiqués. Ces publications sont indiquées dans les tableaux I, II, III, IV et V.

Les taux d'aberrations chromosomiques présentés dans notre étude sont soit ceux indiqués dans les publications, soient ceux calculés à partir des données brutes (nombre de translocations divisé par le nombre de cellules observées). Les valeurs indiquées dans nos tableaux représentent le taux de translocations pour 1000 cellules. Les erreurs associées à ces taux ne sont pas toujours indiquées dans les tableaux I-V car leur calcul diffère d'une étude à l'autre. En effet, dans certains cas les intervalles de confiance sont données et dans d'autres cas les écart-types. Pour comparer le taux de translocations dans une population exposée à celui d'une population non exposée, certaines publications ont utilisé des tests statistiques. Ces tests ne sont pas toujours décrits avec précision et nous n'avons pas refait les calculs. Nous avons toujours rapporté les résultats de significativité de la publication avec en sus le $p$ quand il était mentionné. En ce qui concerne les écarttypes ou les intervalles de confiance, nous avons préféré accorder les fréquences données à l'aide des intervalles de confiance à $95 \%$ selon la distribution de Poisson. Les données nécessaires étaient le nombre de cellules analysées, le nombre de translocations pour les deux groupes étudiés. Toutefois, ces données n'étaient pas présentes dans toutes les publications. C'est pourquoi ces intervalles ne sont pas mentionnés pour toutes les fréquences, notamment au sein des tableaux II et IV.

\section{Résultats}

\subsection{Taux d'aberrations chromosomiques dans la population générale}

Dans la littérature internationale, deux principales publications présentent la variabilité du taux spontané des translocations à partir des résultats obtenus dans plusieurs laboratoires. La plus ancienne regroupe les résultats de laboratoires européens (Whitehouse et al., 2005) et la plus récente regroupe les résultats issus d'un grand nombre de laboratoires dans le monde (Sigurdson et al., 2008). Les données de l'étude européenne sont elles-mêmes incluses dans les données de l'étude mondiale.

D'après le tableau I, le taux de translocations constaté varie d'un facteur 3 entre le laboratoire présentant le taux le plus bas et celui montrant le taux le plus élevé 


\section{TABLEAU I}

Effet laboratoire présentant le taux de translocations minimum et maximum observé. Études de Whitehouse et Sigurdson. Taux pour 1000 cellules observées.

Minimum and maximum rate observed in laboratories studied by the studies of Whitehouse and Sigurdson. Rate given for 1000 observed cells.

\begin{tabular}{|lccc}
\hline $\begin{array}{c}\text { Référence de la } \\
\text { publication }\end{array}$ & $\begin{array}{c}\text { Taux minimum de } \\
\text { translocations observé } \\
\text { pour un laboratoire }\end{array}$ & $\begin{array}{c}\text { Taux maximum de } \\
\text { translocations observé } \\
\text { pour un laboratoire }\end{array}$ & $\begin{array}{c}\text { Nombre d'individus total } \\
\text { dans l'étude }\end{array}$ \\
\hline Whitehouse et al., $2005 *$ & $\begin{array}{c}2,98[2,09-4,14] \\
(22 \text { personnes })\end{array}$ & $\begin{array}{c}8,90[7,00-11,16] \\
(18 \text { personnes })\end{array}$ & 385 \\
Sigurdson et al., $2008 *$ & $\begin{array}{c}2,5[1,8-3,4] \\
(35 \text { personnes })\end{array}$ & $\begin{array}{c}22,5[20,2-25,1] \\
(113 \text { personnes })\end{array}$ \\
\hline
\end{tabular}

* Taux calculé à partir des données de la publication pour chaque laboratoire. Ce taux est obtenu à partir du nombre de translocations total toutes classes d'âge confondues divisé par le nombre total de cellules observé. L'intervalle de confiance à $95 \%$ ([ ]) est calculé à partir du nombre de translocations observées et selon la table de la loi de Poisson. Dans la publication de Sigurdson et al. (2008), le taux est donné pour 100 cellules observées. Dans celle de Whitehouse et al. (2005), il est donné pour 1000 cellules. Les données sont rapportées à l'ensemble du génome selon la formule de Lucas. ( ) Nombre de personnes analysé dans le laboratoire.

au sein de l'étude de Whitehouse et al. (2005) et d'un facteur 10 dans celle de Sigurdson et al. (2008). En outre, l'étude de Sigurdson et al. (2008) met en évidence une variation significative entre les 4 principales zones géographiques (Europe de l'Ouest, Europe Centrale et de l'Est, Asie, Amérique du Nord). En revanche, aucun effet d'ethnie n'est observé mais plutôt un effet inter-laboratoires. Il apparaît alors essentiel, lorsqu'on étudie des facteurs ayant une influence sur le taux de translocations, de comparer des données uniquement issues du même laboratoire. C'est pourquoi chaque laboratoire doit avoir sa propre courbe de référence afin d'éliminer tout impact de la méthodologie sur le résultat.

\subsection{Effet de l'âge}

De nombreuses publications attestent que le taux de translocations augmente significativement avec l'âge (Darroudi, 2000 ; Edwards et al., 2005 ; Pressl et al., 1999 ; Ramsey et al., 1995 ; Sigurdson et al., 2008 ; Sorokine-Durm et al., 2000a ; Whitehouse et al., 2005). Ces études peuvent être regroupées en deux groupes : celles regroupant les données issues de plusieurs laboratoires pour avoir plus de poids statistique (Sigurdson et al., 2008; Sorokine-Durm et al., 2000b; Whitehouse et al., 2005), et celles qui présentent les données d'un seul laboratoire (Darroudi et Natarajan, 2000 ; Pressl et al., 1999 ; Ramsey et al., 1995) (Tab. II).

Dans le cas des études sur une seule population, l'augmentation des translocations en fonction de l'âge est montrée même si une analyse statistique ne l'appuie pas toujours (Darroudi et Natarajan, 2000 ; Pressl et al., 1999 ; Ramsey et al., 1995). Une augmentation plus forte est prouvée à partir de 60 ans. En effet 
TABLEAU II

Comparaison des taux de translocations publiés pour différentes tranches d'âge. Les taux sont donnés pour 1000 cellules observées.

Comparison of translocation rate between young and aged people. Rate given for 1000 observed cells.

\begin{tabular}{|c|c|c|c|c|}
\hline $\begin{array}{c}\text { Référence de la } \\
\text { publication }\end{array}$ & $\begin{array}{c}\text { Taux de translocations } \\
\text { pour les personnes les } \\
\text { plus jeunes }\end{array}$ & $\begin{array}{c}\text { Taux de translocations } \\
\text { pour les personnes les } \\
\text { plus âgées }\end{array}$ & $\begin{array}{c}\text { Facteur } \\
\text { d'augmentation * } \\
\text { (Significativité) }\end{array}$ & $\begin{array}{c}\text { Nombre } \\
\text { d'individus total } \\
\text { dans l'étude }\end{array}$ \\
\hline $\begin{array}{l}\text { Sigurdson } \\
\text { et al., } 2008 \text { a b }\end{array}$ & $\begin{array}{l}0,4[0,3-0,5] \\
\quad(0 \text { an })\end{array}$ & $\begin{array}{c}18,1[13,9-23,6] \\
(80 \text { ans et }+)\end{array}$ & $46 \quad(p<0,001)$ & $\begin{array}{l}296(0) \\
25(80+)\end{array}$ \\
\hline $\begin{array}{l}\text { Whitehouse } \\
\text { et al., 2005 } \mathrm{bc}\end{array}$ & $\begin{array}{c}0,10[0,00-0,54] \\
(0 \text { an })\end{array}$ & $\begin{array}{c}13,41[11,68-15,41] \\
(70-79)\end{array}$ & $160 \quad(p<0,05)$ & $\begin{array}{c}30 \text { (0 an }) \\
41 \text { (70-79 ans) }\end{array}$ \\
\hline $\begin{array}{l}\text { Ramsey et al., } \\
1995^{\mathrm{e}}\end{array}$ & $1,18^{\mathrm{d}}$ (Sang de cordon) & $12,53^{d}(50$ ans et +$)$ & $10,6^{\mathrm{f}} \quad(p<0,0001)$ & $\begin{array}{l}14 \text { (cordon) } \\
37(50+)\end{array}$ \\
\hline $\begin{array}{l}\text { Darroudi et } \\
\text { Natarajan, } 2000\end{array}$ & Non précisé & Non précisé & $1,5^{\mathrm{f}}$ & $\begin{array}{c}18 \text { (21 à } 30) \\
17 \text { (> } 51 \text { ans })\end{array}$ \\
\hline $\begin{array}{l}\text { Pressl et al., } \\
1999 \mathrm{~g}\end{array}$ & $1,29[0,86-1,85]$ & $3,52[2,80-4,35]$ & 2,7 & $\begin{array}{c}9(20-29) \\
6(60+)\end{array}$ \\
\hline
\end{tabular}

${ }^{a}$ Moyenne des fréquences de translocation et intervalle de confiance à $95 \%$ pour 100 cellules équivalentes de tous les laboratoires entre le groupe le plus jeune et le groupe le plus âgé. ${ }^{b}$ Les taux sont données en équivalents génome par la formule de Lucas. ${ }^{\mathrm{c}}$ Fréquence de translocations de tous les laboratoires pour 1000 cellules équivalentes entre le groupe le plus jeune et le groupe le plus âgé. ${ }^{d}$ Les valeurs présentées dans la publication ont été divisées par deux pour pouvoir être comparées aux autres données publiées. Ceci résulte de la façon de compter une translocation impliquant deux chromosomes, soit comme une translocation soit comme deux translocations, deux chromosomes étant porteur de la translocation. ${ }^{\mathrm{e}}$ Valeurs données pour 100 cellules observées au sein de la publication. ${ }^{\mathrm{f}}$ Facteur d'augmentation donné dans la publication. ${ }^{\mathrm{g}}$ Fréquence de translocations non génomique équivalent mais le facteur d'augmentation reste le même. L'intervalle de confiance à $95 \%$ ([ ]) est calculé à partir du nombre de translocations observées et selon la table de la loi de Poisson. * Ratio du Taux de translocation de la population âgée sur taux de translocation de la population jeune.

le taux est multiplié par 3 entre 40 ans et 60 ans (Pressl et al., 1999) et par 3,5 entre le groupe 19-49 ans et le groupe 50 ans et plus (Ramsey et al., 1995). Il faut noter que dans la publication de Ramsey et al. (1995), les taux sont donnés en nombre de cassures par cellule, donc nous avons divisé par 2 les valeurs de fréquence de translocations données puisque il est convenu qu'une translocation compte pour 2 cassures. Cependant, la décision d'utiliser les cassures comme mesure en cytogénétique peut mener à une grande variabilité dans le comptage (Pressl et al., 1999). Ceci peut expliquer le facteur d'augmentation assez élevé observé dans l'étude de Ramsey et al.

L'étude la plus importante regroupe les données issues de 16 laboratoires et totalise 1933 individus (Sigurdson et al., 2008). Nous avons réparti les données des 1933 individus en 3 parties en fonction de l'âge (moins de 25 ans, de 25 à 59 ans et plus de 60 ans). Dans cette étude internationale, le facteur d'accroissement des 
translocations par l'effet âge est significatif quel que soit le laboratoire. Les taux de translocations augmentent de la manière la plus significative entre la classe 0 an et la classe 20-29 ans (d'un facteur 2 à 3) et entre la classe 40-49 et la classe 6079 ans (d'un facteur 2). Sachant que le nombre de translocations pour 1000 cellules observées chez les nouveau-nés est voisin de 0 (Tucker, 2008), une quantité de translocations non négligeable est engendrée entre 0 et 20 ans. Les augmentations se produisant entre la naissance et la vingtaine par une activité clastogène interne avec l'âge, telle que les espèces oxygénées radicalaires, peut être possible mais une telle relation avec l'induction d'échanges chromosomiques n'a pas été établi (Beskid et al., 2006). Cela confirme l'importance du choix de la population la plus jeune dans la comparaison avec une population la plus âgée. En effet, l'augmentation du taux de translocation est souvent significative quand le groupe le plus jeune est composé de nouveaux-nés (Ramsey et al., 1995 ; Sigurdson et al., 2008 ; Whitehouse et al., 2005). L'étude européenne de Whitehouse et al. (2005) montre également une augmentation du taux de translocations avec l'âge pour tous les laboratoires avec une augmentation plus significative après 60 ans (Whitehouse et al., 2005). Ceci est confirmé dans l'étude internationale par le taux de translocations très élevé observé dans les laboratoires de l'Europe de l'Est et Centrale (Sigurdson et al., 2008). Ce taux est intermédiaire dans les laboratoires d'Asie et d'Amérique du nord et enfin plus faible pour ceux d'Europe de l'Ouest, mais ce dernier résultat est dû aux taux très faible enregistré à tous les âges dans un seul laboratoire. Globalement l'âge est le facteur qui a un fort impact sur le nombre de translocations ( $p<0,001$ ) (Sigurdson et al., 2008). Il est indispensable de le prendre en compte dans l'estimation de dose en cas d'accident d'irradiation, en comparant le taux de translocations observées dans les lymphocytes de l'individu exposé au taux de translocations de la population non-exposée compte tenu de son âge par un test ANOVA sur la comparaison entre 2 fréquences (CETAMA, 1998). Il est donc nécessaire que chaque laboratoire établisse le taux de translocations en fonction de classes d'âge croissantes au sein d'une population non exposée.

\subsection{Effet sexe}

Parmi les rares publications qui étudient l'effet sexe sur le taux de translocations seule celle de Whitehouse met en évidence un taux de translocations plus élevé significativement chez les hommes que chez les femmes pour la classe d'âge 3039 ans $(p<0,05)$. Pour les autres classes d'âge, aucune différence n'est prouvée entre le taux des translocations chez les femmes et celui des hommes (Whitehouse et al., 2005) (Tab. III). D'une manière générale, les autres études ne mettent pas en évidence de différence significative sur le taux de translocations selon le genre des individus (Pressl et al., 1999 ; Ramsey et al., 1995 ; Sigurdson et al., 2008), même par classe d'âge. 
TABLEAU III

Synthèse des publications étudiant l'effet du sexe sur le taux de translocations. Les taux sont donnés pour 1000 cellules observées.

Summary of publications investigating the effect of gender on translocation rate. Rate given for 1000 observed cells.

\begin{tabular}{|c|c|c|c|c|}
\hline $\begin{array}{c}\text { Référence de la } \\
\text { publication }\end{array}$ & $\begin{array}{c}\text { Taux de } \\
\text { translocations } \\
\text { chez les femmes }\end{array}$ & $\begin{array}{c}\text { Taux de } \\
\text { translocations } \\
\text { chez les hommes }\end{array}$ & $\begin{array}{c}\text { Facteur } \\
\text { d'augmentation } \\
\text { (Significativité) }\end{array}$ & $\begin{array}{l}\text { Nombre d'individus total } \\
\text { dans l'étude }\end{array}$ \\
\hline \multirow{2}{*}{$\begin{array}{l}\text { Whitehouse et al., } \\
2005^{\text {a }}\end{array}$} & $\begin{array}{c}3,54^{\mathrm{b}} \\
{[2,60-4,71]}\end{array}$ & $\begin{array}{c}6,14^{\mathrm{b}} \\
{[5,05-7,39]}\end{array}$ & $1,7^{*} \quad(p<0,05)$ & $\begin{array}{l}\text { 30-39 ans : } 15 \text { femmes } \\
25 \text { hommes }\end{array}$ \\
\hline & $6,0^{\mathrm{c}}[5,4-6,7]$ & $7,9^{\mathrm{c}}[7$, & $1,3^{*} \quad \mathrm{~N}$ & $\begin{array}{l}\text { Toutes classes âges: } \\
109 \text { femmes } 246 \text { hommes }\end{array}$ \\
\hline & $1,5[1,2-1,8]$ & $1,7[1,4-2,0]$ & NS & \\
\hline $\begin{array}{l}\text { Sigurdson et al., } \\
2008 \text { a c d }\end{array}$ & $3,18[3,00-3,37]$ & $5,52[5,30-5,74]$ & $1,7 * \mathrm{NS}(p=0,10)$ & \\
\hline \multicolumn{5}{|c|}{$\begin{array}{l}{ }^{\mathrm{a}} \text { Les taux sont données en équivalents génome par la formule de Lucas. }{ }^{\mathrm{b}} \text { Fréquence des taux de translocation de } \\
\text { tous les laboratoires pour } 1000 \text { cellules équivalentes selon le genre pour la classe d'âge } 30-39 \text { ans. }{ }^{\mathrm{c}} \text { Taux calculé à } \\
\text { partir des données de la publication à partir du nombre de translocations observé sur le nombre total de cellules } \\
\text { analysées ramené à } 1000 \text { cellules. d Valeurs données pour } 100 \text { cellules observées au sein de la publication. } \\
{ }^{\mathrm{e}} \text { Fréquence de translocations non génomique équivalent mais le facteur d'augmentation reste le même. } \\
\text { L'intervalle de confiance à } 95 \% \text { ([ ]) est calculé à partir du nombre de translocations observées et selon la table de } \\
\text { la loi de Poisson. NS : difference non significative. * Ratio du Taux de translocation chez les hommes sur taux de } \\
\text { translocation chez les femmes. }\end{array}$} \\
\hline
\end{tabular}

\subsection{Effet du tabagisme}

L'effet du tabagisme sur la production d'aberrations chromosomiques est souvent analysé parallèlement à l'influence d'autres agents génotoxiques. Ainsi, les résultats obtenus sont souvent contradictoires puisque l'effet tabac sur les fréquences de translocations peut être masqué ou bien renforcé par les effets des autres agents. La majorité des études présentées ne montrent pas d'augmentation significative du taux de translocations (Tab. IV).

Sur les 10 publications qui étudient l'impact du tabac sur le nombre de translocations, sept ne montrent pas d'augmentation significative du taux de translocations après une consommation excessive de tabac (plus de 20 cigarettes par jour) (Beskid et al., 2007 ; Maeng et al., 2004 ; Pressl et al., 1999; Tawn et Cartmell, 1989 ; Tucker et al., 2003 ; Van Diemen et al., 1995 ; Whitehouse et al., 2005). La consommation de cigarettes par jour varie selon le terme employé. Le terme fumeur s'applique pour une consommation de 10 à 20 cigarettes par jour et le terme « gros fumeur » est utilisé pour les personnes dépassant les 20 cigarettes par jour, voire plus de 30 cigarettes par jour.

L'effet non significatif est souvent dû à l'effet masquant de l'âge (Whitehouse et al., 2005), mais d'autres publications ne l'ont pas observé (Tawn et Cartmell, 1989). À la trentaine et la soixantaine, le taux de translocation est plus élevé chez 
TABLEAU IV

Synthèse des publications étudiant l'effet du tabac sur le taux de translocations. Les taux sont donnés pour 1000 cellules observées.

Summary of publications investigating the effect of smoking on translocation rate. Rate given for 1000 observed cells.

\begin{tabular}{|c|c|c|c|c|c|}
\hline $\begin{array}{c}\text { Référence de la } \\
\text { publication }\end{array}$ & $\begin{array}{c}\text { Taux de } \\
\text { translocations chez } \\
\text { les non-fumeurs }\end{array}$ & $\begin{array}{c}\text { Taux de } \\
\text { translocations chez } \\
\text { les fumeurs }\end{array}$ & \multicolumn{2}{|c|}{$\begin{array}{c}\text { Facteur } \\
\text { d'augmentation } \\
\text { (Significativité) }\end{array}$} & $\begin{array}{c}\text { Nombre } \\
\text { d'individus total } \\
\text { dans l'étude } \\
\text { non-fumeurs }(\mathrm{NF}) \\
\text { fumeurs }(\mathrm{F})\end{array}$ \\
\hline $\begin{array}{l}\text { Tawn et Cartmel, } \\
1989^{\text {a b }}\end{array}$ & $4,2[1,4-9,7]$ & $5,0[1,8-10,9]$ & $1,2 *$ & (NS) & $\begin{array}{l}12 \mathrm{NF} \\
12 \mathrm{~F}\end{array}$ \\
\hline $\begin{array}{l}\text { Van Diemen et al., } \\
1995^{\text {c d }}\end{array}$ & 8,0 & 8,3 & $1,04 *$ & (NS) & $\begin{array}{c}30 \mathrm{NF} \\
12 \mathrm{~F}\end{array}$ \\
\hline $\begin{array}{l}\text { Pressl et al., } \\
1999^{\text {a c h }}\end{array}$ & $2,05[1,67-2,48]$ & $2,01[1,64-2,44]$ & $1 *$ & (NS) & $\begin{array}{c}18 \mathrm{NF} \\
18 \mathrm{~F}\end{array}$ \\
\hline $\begin{array}{l}\text { Tucker et al., } \\
2003 \text { cd }\end{array}$ & $3,70 \pm 0,54(\mathrm{SE})$ & $5,11 \pm 0,64(\mathrm{SE})$ & $1,4 *$ & (NS) & $\begin{array}{l}20 \mathrm{NF} \\
26 \mathrm{~F}\end{array}$ \\
\hline $\begin{array}{l}\text { Whitehouse et al., } \\
2005 \text { a c }\end{array}$ & $6,84[6,36-7,36]$ & $8,15[7,39-8,98]$ & $1,2 *$ & (NS) & $\begin{array}{l}216 \mathrm{NF} \\
40 \mathrm{GF}\end{array}$ \\
\hline $\begin{array}{l}\text { Maeng et al., } \\
2004^{\text {a c }}\end{array}$ & $4,58[1,84-9,44]$ & $5,38[2,69-9,63]$ & $1,17 *$ & $(\mathrm{NS}) * *$ & $\begin{array}{l}22 \mathrm{NF} \\
60 \mathrm{~F}\end{array}$ \\
\hline $\begin{array}{l}\text { Beskid et al., } \\
2007^{\mathrm{cd}}\end{array}$ & $10,4 \pm 10,3$ & $12,9 \pm 13,4$ & $1,24^{*}$ & $(\mathrm{NS})^{* *}$ & $\begin{array}{l}84 \mathrm{NF} \\
35 \mathrm{~F}\end{array}$ \\
\hline $\begin{array}{l}\text { Pluth et al., } 2000^{\mathrm{cd}} \\
\text { fréquence } \pm \mathrm{SE} \text { (SD } \\
\text { donné dans l'article) }\end{array}$ & $\begin{array}{c}1,1 \pm 0,7 \\
(\mathrm{SE}=\mathrm{SD} / \sqrt{\mathrm{N}}: \\
0,7=2,1 / \sqrt{8})\end{array}$ & $\begin{array}{c}1,1 \pm 0,4 \\
(\mathrm{SE}=\mathrm{SD} / \sqrt{\mathrm{N}}: \\
0,4=0,8 / \sqrt{4})\end{array}$ & $1 *$ & $(\mathrm{NS}) * * *$ & $\begin{array}{l}8 \mathrm{NF} \\
4 \mathrm{~F}\end{array}$ \\
\hline $\begin{array}{l}\text { Ramsey et al., } \\
1995^{\text {ac d g }} \\
\text { Etude nouveau-nés }\end{array}$ & 1,97 & 2,87 & $1,5^{*}(p$ & $=0,029)^{* *}$ & $\begin{array}{l}8 \mathrm{NF} \\
6 \mathrm{~F}\end{array}$ \\
\hline $\begin{array}{l}\text { Bothwell et al., } \\
2000^{\mathrm{c} \mathrm{e}}\end{array}$ & $\begin{array}{l}11,45 \pm 1,29 \\
{[9,06-14,27]}\end{array}$ & $\begin{array}{c}15,99 \pm 1,15 \\
{[13,81-18,42]}\end{array}$ & $1,4 *(p$ & $0,05)^{* * * *}$ & $\begin{array}{l}20 \mathrm{NF} \\
35 \mathrm{~F}\end{array}$ \\
\hline $\begin{array}{l}\text { Sigurdson et al., } \\
2008_{\text {a c f }}\end{array}$ & $3,99[3,83-4,16]$ & $5,01[4,78-5,25]$ & $1,25^{*}$ & $p<0,001)$ & $\begin{array}{c}804 \mathrm{NF} \\
596 \mathrm{~F}\end{array}$ \\
\hline
\end{tabular}

F : Fumeurs, GF : gros fumeurs, NF : non-fumeurs. NS : Difference non significative. ${ }^{\mathrm{a}}$ Taux calculé à partir des données de la publication : obtenu par le ratio du nombre de translocation par le nombre de cellules observées. ${ }^{\mathrm{b}}$ Marquage des chromosomes au Giemsa. Taux de translocation donné pour 1200 cellules dans la publication. ${ }^{\mathrm{c}}$ Les taux sont données en équivalents génome par la formule de Lucas. ${ }^{\mathrm{d}}$ Fréquence de translocation donnée pour 100 cellules observées au sein de la publication. ${ }^{\mathrm{e}}$ Fréquence de translocation pour 1000 cellules équivalentes entre le groupe «gros-fumeur» et le groupe non-fumeur. ${ }^{\mathrm{f}}$ Fréquence de translocation pour 1000 cellules équivalentes de tous les laboratoires entre le groupe non-fumeur et le groupe fumeur. ${ }^{g}$ Les valeurs présentées dans la publication ont été divisées par deux pour pouvoir être comparées aux autres données publiées. Ceci résulte de la façon de compter une translocation impliquant deux chromosomes, soit comme une translocation soit comme deux translocations, deux chromosomes étant porteur de la translocation. ${ }^{\mathrm{h}}$ Fréquence de translocations non génomique équivalent mais le facteur d'augmentation reste le même. L'intervalle de confiance à $95 \%$ ([ ]) est calculé à partir du nombre de translocations observées et selon la table de la loi de Poisson. * Ratio du Taux de translocation chez les fumeurs sur taux de translocation chez les non-fumeurs. ** Test Mann-Whitney. *** Test Wilcoxon. $* * * *$ Test $\mathrm{X}^{2}$. 
les gros fumeurs par rapport à celui des non-fumeurs, mais en aucun cas cela n'est significatif (Whitehouse et al., 2005). De même le tabac modifie significativement le taux de translocations chez les fumeurs chroniques par rapport à celui des nonfumeurs $(p<0,001)$, notamment après 60 ans (Sigurdson et al., 2008). En procédant à la modélisation multidimensionnelle, l' ajustement statistique des autres facteurs a également permis aux auteurs d'identifier un effet tabac. Cependant, les auteurs précisent qu'ils ne peuvent spéculer que sur un effet du tabagisme biologiquement significatif (Sigurdson et al., 2008). Au sein des 2 autres publications montrant une augmentation significative du nombre de translocations sous l'effet du tabac, l'une montre l'effet additionnel du tabagisme sur le taux de translocations engendré par les rayonnements ionisants. En effet, elle a été réalisée au sein de retraités ayant été exposés professionnellement à des doses annuelles de rayonnement ionisants (<50 mSv) (Bothwell et al., 2000). Cependant, ni l'effet âge ni la consommation ne sont mentionnée dans cet article. L'autre étude montre une augmentation du nombre de translocations chez les nouveaux-nés dont la mère a fumé pendant la grossesse (groupe exposé) par rapport à celui de nouveaux-nés dont les parents ne fument pas (groupe témoin) (Ramsey et al., 1995). La consommation d'alcool n'est pas précisée. La différence est d'autant plus significative que le nombre de translocations pour 1000 cellules observées chez les nouveau-nés est voisin de 0 (Tucker, 2008). En revanche, cette augmentation significative des translocations n'est pas montrée chez des nouveau-nés dont la mère a uniquement fumé (non consommatrice d'alcool en plus) pendant la grossesse par rapport aux translocations des nouveau-nés de mères non-fumeuses, ni consommatrice d'alcool (Pluth et al., 2000). Dans ce cas, l'exposition des nouveau-nés s'apparenterait plutôt au tabagisme passif qui n'engendrerait pas d' aberrations chromosomiques (HusgafvelPursiainen, 2004). À ce jour, aucune étude n'a mis en évidence une augmentation du taux de translocations due au tabagisme passif chez les adultes.

Dans la plupart des publications analysées, le tabac ne provoque pas d'augmentation significative du taux de translocations. Dans l'article Sigurdson et al., l'effet tabac est fortement lié à l'effet âge puisqu' après ajustement par l'âge, l'augmentation des translocations n'est plus significative.

\subsection{Effet de la consommation d'alcool}

La consommation des sujets alcooliques va de $60 \mathrm{~g}$ d'éthanol par jour à plus de $200 \mathrm{~g}$ d'éthanol par jour, sachant qu'un verre d'alcool servi dans les bars correspond en moyenne à $10 \mathrm{~g}$ d'éthanol. Deux études sur les effets de la consommation d'alcool ont montré une augmentation significative du taux des translocations chez des alcooliques chroniques (Badr et Hussain, 1982 ; Burim et al., 2004) (Tab. V). Cependant, cette consommation d'alcool est souvent liée à la consommation 
TABLEAU V

Synthèse des publications étudiant l'effet de l'alcool sur le taux de translocations. Les taux sont donnés pour 1000 cellules observées.

Summary of publications investigating the effect of alcoholism on translocation rate. Rate given for 1000 observed cells.

\begin{tabular}{|ccccc}
\hline $\begin{array}{c}\text { Référence de la } \\
\text { Publication }\end{array}$ & $\begin{array}{c}\text { Taux de } \\
\text { translocations dans la } \\
\text { population contrôle }\end{array}$ & $\begin{array}{c}\text { Taux de translocations } \\
\text { chez les alcooliques } \\
\text { chroniques }\end{array}$ & $\begin{array}{c}\text { Facteur } \\
\text { d'augmentation }\end{array}$ & $\begin{array}{c}\text { Nombre } \\
\text { d'individus total } \\
\text { dans l'étude }\end{array}$ \\
\hline Badr et Hussain, 1982 & $0,0^{\mathrm{a}}[0-0,9]$ & $5,2^{\mathrm{a}}[2,8-8,9]$ & $-*(p<0,05)$ & $\begin{array}{c}23 \text { alcooliques } \\
50 \text { contrôles }\end{array}$ \\
Burim et al., 2004 ${ }^{\mathrm{b} \text { c }}$ & $1,98[0,56-5,25]$ & $7,90[4,29-12,19]$ & $3,95^{*}(p<0,05)^{* *} \begin{array}{c}6 \text { alcooliques } \\
\text { chroniques } \\
6 \text { contrôles }\end{array}$ \\
\hline
\end{tabular}

\footnotetext{
${ }^{\mathrm{a}}$ Marquage des chromosomes au Giemsa. ${ }^{\mathrm{b}}$ Les taux sont données en équivalents génome par la formule de Lucas. ${ }^{c}$ Fréquences de translocation donnée pour 1000 cellules observées au sein de la publication. L'intervalle de confiance à $95 \%$ ([ ]) est calculé à partir du nombre de translocations observées et selon la table de la loi de Poisson. * Ratio du Taux de translocation chez les fumeurs sur taux de translocation chez les non-fumeurs. ** Test Fisher. $* * * *$ Test $\mathrm{X}^{2}$.
}

d'autres agents liés au mode de vie, tels que tabac, alcools frelatés (présence de métaux lourds ou d'agents chimiques mutagènes) ou impropres à la consommation (eau de Cologne) (Badr et Hussain, 1982). Par conséquent, un effet synergique de l'alcool associé à d'autres facteurs comportementaux peut être à l'origine de cette augmentation. Ceci a été montré précédemment sur une étude avec le tabagisme et les rayonnements ionisants (Bothwell et al., 2000). Mais cet effet est difficile à mettre en évidence pour l'alcool puisqu'il ne semble pas exister d'études mettant en évidence l'effet de l'alcoolisme exempt d'autres addictions.

\section{Conclusion}

Les translocations sont les aberrations chromosomiques utilisées en cas d'accident d'irradiation afin d'estimer une dose de rayonnements ionisants reçue plusieurs années précédant l'analyse. Toutefois, afin que les translocations puissent être utilisées en dosimétrie rétrospective, il est nécessaire d'évaluer leur spécificité. Pour cela, une étude bibliographique de l'impact de certains facteurs biologique et comportementaux sur la production des translocations est nécessaire. Dans cet objectif, cet article fait la synthèse de publications étudiant l'impact de facteurs tels que l'âge, le sexe, le tabac et l'alcool sur la production des translocations. Il est important de soulever le fait que le nombre de publications qui étudie l'impact d'un facteur biologique ou comportemental donné est assez faible, le nombre d'études n'excède pas 10 pour chaque facteur.

L'effet Âge est le facteur majoritaire générant des translocations, essentiellement à partir de 60 ans. Il est alors important de prendre en compte ce facteur et de 
comparer le nombre de translocations observées dans les lymphocytes du patient au taux attendu de translocations compte tenu de son âge. Il est donc indispensable que chaque laboratoire établisse son bruit de fond en fonction des différentes classes d'âge.

En revanche, le bruit de fond naturel ne peut expliquer à lui seul l'augmentation due à l'âge. Il y a donc d'autres facteurs tels que ceux auxquels l'individu s'expose comme les agents liés au style de vie (Whitehouse et al., 2005). Ainsi, le tabac seul a peu d'influence sur le taux de translocations. En revanche, la consommation d'alcools forts engendre une augmentation des translocations, notamment quand elle est accompagné par la consommation d'autres agents toxiques (métaux lourds, tabac). Par conséquent, d'autres études doivent être réalisées afin de connaitre l'effet sur le taux de translocations de l'alcool seul. Ainsi nous pourrions établir une courbe dose effet du taux de translocations en fonction de la consommation alcoolique.

Après avoir établi le taux de translocation engendré par les facteurs étudiés dans cette revue bibliographique, le but est de comparer ces taux à ceux induits par une exposition aux rayonnements ionisants. La figure 3 illustre l'impact de chaque agent sur le taux des translocations en fonction de l'exposition et des études. Pour chaque étude le taux de translocations pour 1000 cellules de la population contrôle est indiqué en $\$$ et celui de la population exposée en 目. En outre, les taux de translocations obtenus dans notre laboratoire après irradiation in vitro d'échantillons de sang à des doses de 0,$2 ; 0,5$ et 0,7 Gy ont été aussi représentés sur cette figure. En comparant le taux induit par une dose d'irradiation de 0,2 Gy, le taux de translocations des fumeurs est faible. En effet, celui des non fumeurs est presque similaire à celui des fumeurs. Il n'y a que l'étude de Bothwell qui pourrait prétendre masquer une exposition à 0,5 Gy mais nous rappelons que cette étude concernait des personnes ayant été exposé aux rayonnements ionisants. En revanche, une consommation importante d'alcool est susceptible de masquer une dose d'irradiation de $0,2 \mathrm{~Gy}$ par le taux de translocations généré. Toutefois, elle ne peut masquer le taux induit par une irradiation supérieure à $0,5 \mathrm{~Gy}$.

Par conséquent il serait tout de même utile de connaitre le bruit de fond des translocations engendré par ces deux styles de vie en fonction de leur consommation. Ces données pourraient être prises en compte au sein d'une courbe du taux de translocations en fonction de la quantité d'alcool et de tabac consommée. Comme pour l'âge, en comparant le taux de translocations du patient par rapport au taux engendré par sa consommation tabagique et/ou alcoolique, la sensibilité de la technique FISH pour les translocations serait améliorée. 


\section{IMPACT DES FACTEURS INDIVIDUELS ET ENVIRONNEMENTAUX}

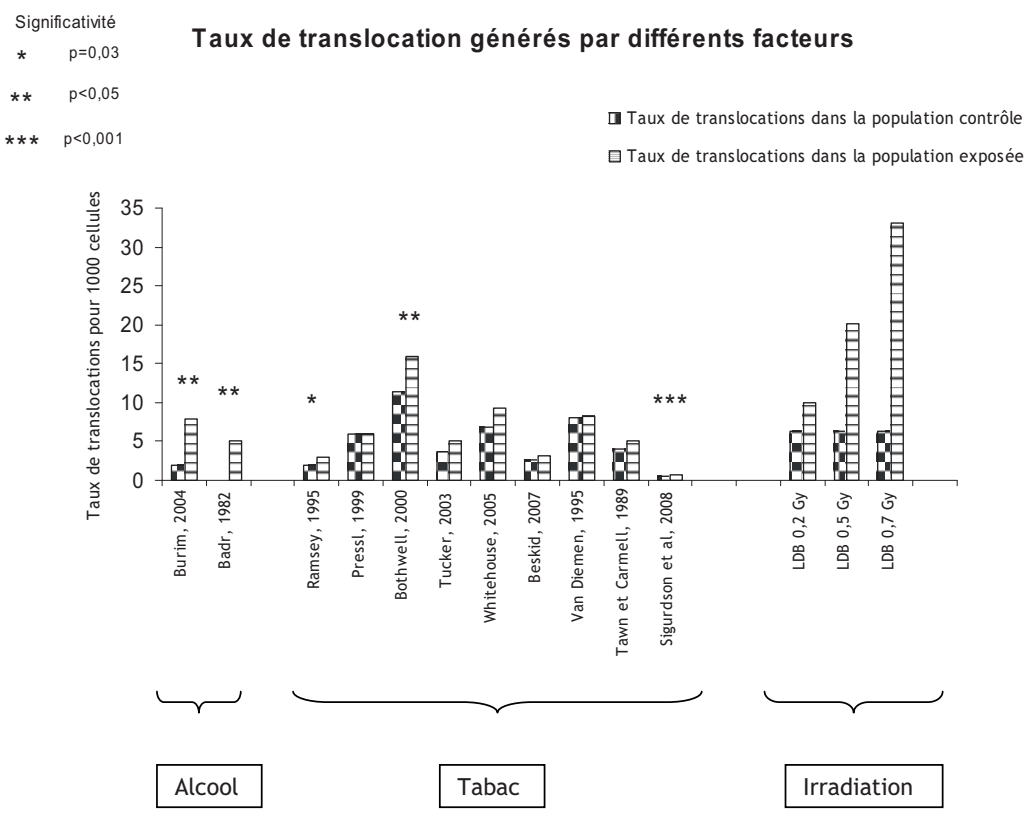

Les taux de translocations sont ceux obtenus dans les publications notées en abscisse $X$. Les taux de translocations de l'irradiation sont ceux obtenus au laboratoire de Dosimétrie Biologique de L'IRSN pour des doses spécifiques.

Figure 3 - Comparaison entre les taux de translocations générées par différents facteurs et le taux de translocations générées par une irradiation in vitro.

Comparison between the translocation rate generated by different factors and that generated by irradiation in vitro.

Ainsi par la connaissance de la fréquence des translocations pouvant être induites par un maximum d'agents, nous pourrions déduire in fine celles réellement radio-induites. Et ainsi augmenter la spécificité de la dosimétrie biologique rétrospective.

Remerciements. Nous remercions vivement Murielle Gautier pour son aide précieuse sans laquelle la recherche bibliographique aurait été laborieuse. Nous remercions également Mr Jean-Marc Bertho pour ses conseils avisés.

\section{RÉFÉRENCES}

Badr F.M., Hussain F.H. (1982) Chromosomal aberrations in chronic male alcoholics, Alcohol. Clin. Exp. Res. 6, 122-129.

Bauchinger M., Braselmann H., Savage J.R.K., Natarajan A.T., Terzoudi G.I., Pantelias G.E., Darroudi F., Figgitt M., Griffin C.S., Knehr S., Okladnikova N.D., Santos S., Snigiryova G. (2001) 


\section{E. GRÉGOIRE $e t a l$.}

Collaborative exercise on the use of FISH chromosome painting for retrospective biodosimetry of Mayak nuclear-industrial personnel, Int. J. Radiat. Biol. 77, 259-267.

Beskid O., Binkova B., Dusek Z., Rossner P., Solansky I., Kalina I., Zidzik J., Popov T.A., Farmer P.B., Sram R.J. (2007) Chromosomal aberrations by fluorescence in situ hybridization (FISH)Biomarker of exposure to carcinogenic PAHs, Mutat. Res. 620, 62-70.

Beskid O., Dusek Z., Solansky I., Sram R.J. (2006) The effects of exposure to different clastogens on the pattern of chromosomal aberrations detected by FISH whole chromosome painting in occupationally exposed individuals, Mutat. Res. 594, 20-29.

Bothwell A.M., Whitehouse C.A., Tawn E.J. (2000) The application of FISH for chromosome aberration analysis in relation to radiation exposure, Radiat. Prot. Dosim. 88, 7-14.

Buckton K.E., Brown W.M., Smith P.G. (1962) Lymphocytes survival in men treated with X-rays for ankylosing spondilitis, Nature 214, 470-473.

Burim R.V., Canalle R., Takahashi C.S., Tavares D.C., Candolo Martinelli A.D.L., Sakamoto-Hojo E.T. (2004) Clastogenic effect of ethanol in chronic and abstinent alcoholics, Mutat. Res. 560, 187-198.

CETAMA (1998) Modélisation et estimation des erreurs de mesure, pp. 127-132. Lavoisier, TEC \& DOC.

Darroudi F. (2000) Use of FISH translocations analyses for retrospective biological dosimetry: How stable are stable chromosome aberrations? Radiat. Prot. Dosim. 88, 101-109.

Darroudi F., Natarajan A.T. (2000) Application of FISH chromosome painting assay for dose reconstruction: State of the art and current views, Radiat. Prot. Dosim. 88, 51-58.

Edwards A.A., Lindholm C., Darroudi F., Stephan G., Romm H., Barquinero J., Barrios L., Caballin M.R., Roy L., Whitehouse C.A., Tawn E.J., Moquet J., Lloyd D.C., Voisin P. (2005) Review of translocations detected by FISH for retrospective biological dosimetry applications, Radiat. Prot. Dosim. 113, 396-402.

Grégoire E., Sorokine-Durm I., Bertho J.M., Jacquet N., Delbos M., Demarquay C., Voisin P., Roy L. (2006) Follow-up of stable chromosomal aberrations in gamma-ray irradiated non-human primates, Int. J. Radiat. Biol. 82, 493-502.

Husgafvel-Pursiainen K. (2004) Genotoxicity of environmental tobacco smoke: a review, Mutat. Res. 567, 427-445.

Lindholm C., Edwards A. (2004) Long-term persistence of translocations in stable lymphocytes from victims of a radiological accident, Int. J. Radiat. Biol. 80, 559-566.

Lucas J.N., Poggensee M., Straume T. (1992) The persistence of chromosome translocations in a radiation worker accidentally exposed to tritium, Cytogenet. Cell Genet. 60, 255-256.

Maeng S.H., Chung H.W., Kim K.J., Lee B.M., Shin Y.C., Kim S.J., Yu I.J. (2004) Chromosome aberration and lipid peroxidation in chromium-exposed workers, Biomarkers 9, 418-434.

Pala F.S., Moquet J.E., Edwards A.A., Lloyd D.C. (2001) In vitro transmission of chromosomal aberrations through mitosis in human lymphocytes, Mutat. Res. 474, 139-146.

Pluth J.M., Ramsey M.J., Tucker J.D. (2000) Role of maternal exposures and newborn genotypes on newborn chromosome aberration frequencies, Mutat. Res. 465, 101-111.

Pressl S., Edwards A., Stephan G. (1999) The influence of age, sex and smoking habits on the background level of fish-detected translocations, Mutat. Res. 442, 89-95.

Ramsey M.J., Moore D.H. 2nd, Briner J.F., Lee D.A., Olsen L., Senft J.R., Tucker J.D. (1995) The effects of age and lifestyle factors on the accumulation of cytogenetic damage as measured by chromosome painting, Mutat. Res. 338, 95-106.

Roy L., Grégoire E., Durand V., Buard V., Delbos M., Paillole N., Sorokine-Durm I., Gourmelon P., Voisin P. (2006) Study of the tools available in biological dosimetry to estimate the dose in cases of accidental complex overexposure to ionizing radiation: the Lilo accident, Int. J. Radiat. Biol. 82, 39-48. 
Sigurdson A.J., Ha M., Hauptmann M., Bhatti P., Sram R.J., Beskid O., Tawn E.J., Whitehouse C.A., Lindholm C., Nakano M., Kodama Y., Nakamura N., Vorobtsova I., Oestreicher U., Stephan G., Yong L.C., Bauchinger M., Schmid E., Chung H.W., Darroudi F., Roy L., Voisin P., Barquinero J.F., Livingston G., Blakey D., Hayata I., Zhang W., Wang C., Bennett L.M., Littlefield L.G., Edwards A.A., Kleinerman R.A., Tucker J.D. (2008) International study of factors affecting human chromosome translocations, Mutat. Res. 652, 112-121.

Sorokine-Durm I., Durand V., Delbos M., Le Baron L., Roy L., Voisin P. (2000a) A French view on FISH painting as a biodosemeter, Radiat. Prot. Dosim. 88, 35-44.

Sorokine-Durm I., Whitehouse C., Edwards A.A. (2000b) The variability of translocation yields amongst control populations, Radiat. Prot. Dosim. 88, 93-99.

Tawn E.J., Cartmell C.L. (1989) The effect of smoking on the frequencies of asymmetrical and symmetrical chromosome exchanges in human lymphocytes, Mutat. Res. 224, 151-156.

Tawn E.J., Whitehouse C.A., Riddell A.E. (2006) FISH chromosome analysis of plutonium workers from the Sellafield nuclear facility, Radiat. Res. 165, 592-597.

Tucker J.D. (2008) Low-dose ionizing radiation and chromosome translocations: a review of the major considerations for human biological dosimetry, Mutat. Res. 659, 211-220.

Tucker J.D., Moore D.H. 2nd, Ramsey M.J., Kato P., Langlois R.G., Burroughs B., Long L., Garry V.F. (2003) Multi-endpoint biological monitoring of phosphine workers, Mutat. Res. 536, 7-14.

Van Diemen P. C. M., Maasdam D., Vermeulen S., Darroudi F., Natarajan A.T. (1995) Influence of smoking habits on the frequencies of structural and numerical chromosomal aberrations in human peripheral blood lymphocytes using the fluorescence in situ hybridization (FISH) technique, Mutagenesis 10, 487-495.

Whitehouse C.A., Edwards A.A., Tawn E.J., Stephan G., Oestreicher U., Moquet J.E., Lloyd D.C., Roy L., Voisin P., Lindholm C., Barquinero J., Barrios L., Caballin M.R., Darroudi F., Fomina J. (2005) Translocation yields in peripheral blood lymphocytes from control populations, Int. J. Radiat. Biol. 81, 139-145. 